\title{
Gene expression analysis: Regulation of key genes associated with mycophenolate mofetil treatment of symptomatic carotid artery stenosis
}

\author{
FENG JIN $^{1 *}$, KAI WANG $^{2 *}$, XIAOCHUAN SUN ${ }^{3}$, ZHANPU ZHANG $^{2}$ and PING HAN ${ }^{1}$ \\ ${ }^{1}$ Department of Radiology, Union Hospital, Tongji Medical College, Huazhong University of Science and Technology, \\ Wuhan, Hubei 430022; ${ }^{2}$ Department of Neurosurgery, The Affiliated Hospital of Inner Mongolia Medical University, Hohhot, \\ Inner Mongolia 010050; ${ }^{3}$ Department of Neurosurgery, The First Affiliated Hospital \\ of Chongqing Medical University, Chongqing 630014, P.R. China
}

Received December 1, 2016; Accepted June 30, 2017

DOI: $10.3892 / \mathrm{mmr} .2017 .7532$

\begin{abstract}
The present study analyzed gene expression arrays to identify differentially-expressed genes (DEGs) between mycophenolate mofetil (MMF)-treated and placebo-treated patients with symptomatic carotid artery stenosis (SCAS). In addition, the key genes involved in the pharmacology of MMF treatment in patients with SCAS were identified. The gene expression dataset was obtained from a Gene Expression Omnibus database, which included 9 MMF-treated and 11 placebo-treated samples. The DEGs were identified between MMF and placebo groups using R software. Furthermore, a protein-protein interaction (PPI) network of the identified DEGS was constructed. The Database for Annotation, Visualization and Integrated Discovery was used to perform Gene Ontology (GO) and Kyoto Encyclopedia of Genes and Genomes (KEGG) pathway enrichment analyses of the 19 most significant DEGs. A total of 210 DEGs between the MMF and placebo groups were screened and their PPI was constructed. GO function analysis revealed that the 19 DEGs were predominantly involved in the tyrosine phosphorylation of signal transducer and activator of transcription-5 protein, which is closely associated with the activation of $\mathrm{T}$ cells. The KEGG pathway analysis suggested that the main metabolic pathways of the 19 DEGs were associated with the pharmacological functioning of MMF in activated T cells. In conclusion, the present study identified numerous key DEGs associated with SCAS, and the results
\end{abstract}

Correspondence to: Dr Ping Han, Department of Radiology, Union Hospital, Tongji Medical College, Huazhong University of Science and Technology, 1277 Jiefang Avenue, Wuhan, Hubei 430022, P.R. China

E-mail: cjr.hanping@vip.163.com

${ }^{*}$ Contributed equally

Key words: carotid artery stenosis, atherosclerosis, genes, pathway analysis, protein-protein interaction network suggested that v-kit Hardy-Zuckerman 4 feline sarcoma viral oncogene homolog and apelin may serve important roles in the MMF treatment of SCAS.

\section{Introduction}

Stroke is the fourth leading cause of mortality and disability in the United States (1), and 10-20\% of strokes are caused by carotid artery disease $(2,3)$. According to epidemiological data, $\sim 7$ million adults in the United States have suffered a stroke (4), of which ischemic strokes account for $~ 90 \%$ (5). In addition, $\sim 10 \%$ of ischemic strokes are caused by carotid artery stenosis (CAS) (6). CAS refers to a narrowing or constriction of the lumen of the carotid artery, usually attributed to atherosclerosis (7). The dynamic and complex process of atherosclerosis remains to be fully understood; however, it is well known that atherosclerosis is characterized by the accumulation of lipid particles and fibrous elements, associated with migration and proliferation of smooth muscle cells, in the large arteries (8-10).

Over the past decade, inflammation and the immune response in atherosclerosis have garnered attention. Previous studies have indicated that low-density lipoprotein particles and their content inside the vessel wall are susceptible to oxidation by free radicals, which may initiate the accumulation and invasion of macrophages, eventually lead to a narrowing of the major arteries $(9,11)$.

During the progression of atherosclerosis, an imbalance between anti-inflammatory and proinflammatory cytokines serves an important role. Previous studies have attempted to identify the immune-associated genes that are involved in atherosclerosis, and achievements have been made $(12,13)$. Superoxide dismutase, which is expressed at higher levels in regions of laminar flow, may combat oxidative stress, and hence limit vascular cell adhesion molecule-1 (VCAM-1) expression and the expression of other inflammatory pathways (12). Nitric oxide arises from endothelial nitric oxide synthase, which is known to be a shear stress-regulated gene, and can inhibit VCAM gene expression through a novel pathway involving inhibition of the activation of nuclear factor- $\kappa \mathrm{B}$, 
the central transcription factor in vascular inflammation (13). In addition, previous studies have reported that interleukin (IL)-35 may upregulate the expression of anti-inflammatory cytokines (14-16). Huang et al (17) investigated the effects of IL-35 on atherosclerosis and hypothesized that IL-35 could be considered a novel target for the treatment of atherosclerosis. However, the majority of genes relevant to atherosclerosis remain unknown.

Mycophenolate mofetil (MMF) is an inhibitor of the enzyme inosine monophosphate dehydroxygenase (IMPDH), and exerts a powerful cytostatic effect on activated $\mathrm{T}$ cells by interfering with their DNA synthesis (18). In the present study, gene expression data were obtained from a Gene Expression Omnibus (GEO) dataset uploaded by van Leuven et al (19), which included 20 carotid endarterectomy samples from patients with CAS ( $>70 \%$ diameter stenosis on angiography) that were randomly assigned to the following treatment groups: Treatment with 1,000 mg MMF $(\mathrm{n}=9)$ or placebo $(n=11)$. Patients were treated with MMF or placebo for $\geq 2$ weeks prior to undergoing carotid endarterectomy (CEA). van Leuven et al (19) reported that the inflammatory process in human atherosclerotic plaques could be modified by short-term treatment with MMF, as determined using mRNA expression profiling. However, this previous study did not analyze the expression data in detail, nor did it determine how MMF functioned in the treatment of symptomatic CAS (SCAS) or the molecular mechanisms of SCAS.

In the present study, the gene expression data were used to identify differentially-expressed genes (DEGs) between MMF-treated and placebo-treated groups, with the aim of identifying potential genes associated with atherosclerosis, which may be considered targets for novel gene therapy. A total of 210 DEGs between the MMF and placebo groups were identified with a threshold of $\mathrm{P}<0.05$. After analyzing the regulatory effects, a regulatory network was constructed based on the DEGs. Subsequently, the data were processed by bioinformatic analyses, including hierarchical clustering, Gene Ontology (GO) terms (molecular function, biological processes and cellular components) analysis and Kyoto Encyclopedia of Genes and Genomes (KEGG) pathway analysis. Finally, the 19 most significant DEGs were screened; the results of these analyses indicated that apelin (APLN) and v-kit Hardy-Zuckerman 4 feline sarcoma viral oncogene homolog (KIT) may be valuable for characterizing the mechanism underlying immunomodulatory therapy in atherosclerosis.

\section{Materials and methods}

Datasets. The GSE13922 original mRNA expression profile used in the present study was downloaded from the National Center of Biotechnology Information GEO (www.ncbi.nlm. nih.gov/geo/). The platform used to analyze these data was the GPL6255 Illumina humanRef-8 v2.0 expression beadchip (Illumina, San Diego, CA, USA).

Identification of DEGs. Background correction and quartile data normalization of the downloaded data were performed using the robust multi-array average (RMA) algorithm (20). Probes without a corresponding gene symbol were filtered and the average value of gene symbols with numerous probes was calculated. The expression profile dataset, including 13,985 genes for the 20 samples, was subsequently obtained. Student's t-test was used to identify DEGs between the MMF and placebo groups using the R software LIMMA package (version 3.3.1; www.r-project.org) (21). Genes with $\mathrm{P}<0.05$ were considered DEGs and genes with $\mathrm{P}<0.01$ were considered the most significant DEGs between the two treatment groups. The most significant DEGs were screened between the MMF and placebo groups using principal components analysis (PCA). Cluster analysis of the most significant DEGs was applied to generate a heat map, which allowed for visualization of the differential gene expression between the two groups.

Protein-protein interaction (PPI) network construction and analysis. The PPI network was constructed from 210 DEGs using the STRING online database (www.string-db.org/). PPI pairs with an interaction score $>0.4$ were used to construct the PPI network. Subsequently, the regulatory relationships between genes were analyzed according to the topological properties of the network. With a threshold of $\mathrm{P}<0.05$ and $\mid \log \mathrm{FCl} \geq 0.5$, the key genes in the network were further screened.

Functional analysis of various DEGs. In order to identify biological functions associated with the pathogenesis of atherosclerosis, bioinformatics analyses, including hierarchical clustering, GO (22) terms (molecular function, biological processes, cellular components) analysis and KEGG (23) pathway analysis, were conducted for the most significant DEGs, using the online Database for Annotation, Visualization and Integrated Discovery tool (24), based on the method of Expression Analysis Systemic Explorer (EASE) test (25). The enrichment threshold was an EASE score of 0.1.

\section{Results}

Data processing. A total of 13,985 genes in 20 samples were obtained after preprocessing of the expression profile. The original expression datasets were processed into expression estimates using the RMA method. As presented in the box plot in Fig. 1, the median of different samples was almost the same following normalization, which indicated a great degree of standardization.

Identification of DEGs. The DEGs between the MMF and placebo groups were identified using LIMMA package (21) in R software. A total of 210 DEGs were identified with the threshold of $\mathrm{P}<0.05$, including 19 most significant DEGs with the threshold of $\mathrm{P}<0.01$ (Table I). Analysis of the most significant DEGs revealed that there were 14 up- and 5 downregulated genes (Fig. 2). As presented in Fig. 3, all of the selected DEGs were screened using PCA to distinguish between the MMF and placebo groups. In the first principal components, $50.82 \%$ of variances were explained, whereas in the second principal component, $9.73 \%$ of variances were explained. In total, the resolution degree of variances was $60.55 \%$.

PPInetwork construction and analysis. A total of 189 PPI pairs were obtained from the STRING database. After wiping out 
Table I. List of the 19 most significant DEGs $(\mathrm{P}<0.01)$.

\begin{tabular}{|c|c|c|c|}
\hline Gene symbol & Gene name & P-value & $\log \mathrm{FC}$ \\
\hline AK4 & Adenylate kinase 4 & 0.0042 & 0.4736 \\
\hline APLN & Apelin & 0.0092 & 0.5261 \\
\hline CKB & Creatine kinase, brain & 0.0011 & 0.9570 \\
\hline CSF2 & Colony stimulating factor 2 (granulocyte-macrophage) & 0.0097 & 0.2979 \\
\hline EBI3 & Epstein-Barr virus induced 3 & 0.0048 & -0.7416 \\
\hline ECE1 & Endothelin converting enzyme 1 & 0.0088 & 0.1873 \\
\hline FAM102A & Family with sequence similarity 102 , member A & 0.0057 & -0.2496 \\
\hline GFRA2 & GDNF family receptor $\alpha 2$ & 0.0068 & 0.7860 \\
\hline GPM6B & Glycoprotein M6B & 0.0064 & 0.7289 \\
\hline HDC & Histidine decarboxylase & 0.0045 & 0.9700 \\
\hline KIT & V-Kit Hardy-Zuckerman 4 feline sarcoma viral oncogene homolog & 0.0018 & 0.6283 \\
\hline LMO4 & LIM domain only 4 & 0.0037 & 0.3623 \\
\hline MGST2 & Microsomal glutathione S-transferase 2 & 0.0049 & -0.3100 \\
\hline MRPL30 & Mitochondrial ribosomal protein $\mathrm{L} 30$ & 0.0031 & 0.1626 \\
\hline NSDHL & NAD(P) dependent steroid dehydrogenase-like & 0.0082 & 0.3269 \\
\hline POLR2I & Polymerase (RNA) II (DNA directed) polypeptide I, $14.5 \mathrm{kDa}$ & 0.0053 & 0.2292 \\
\hline RRM1 & Ribonucleotide reductase catalytic subunit M1 & 0.0094 & 0.2653 \\
\hline SCIN & Scinderin & 0.0022 & -0.9358 \\
\hline WDR41 & WD repeat domain 41 & 0.0042 & 0.4693 \\
\hline
\end{tabular}

FC, fold change.

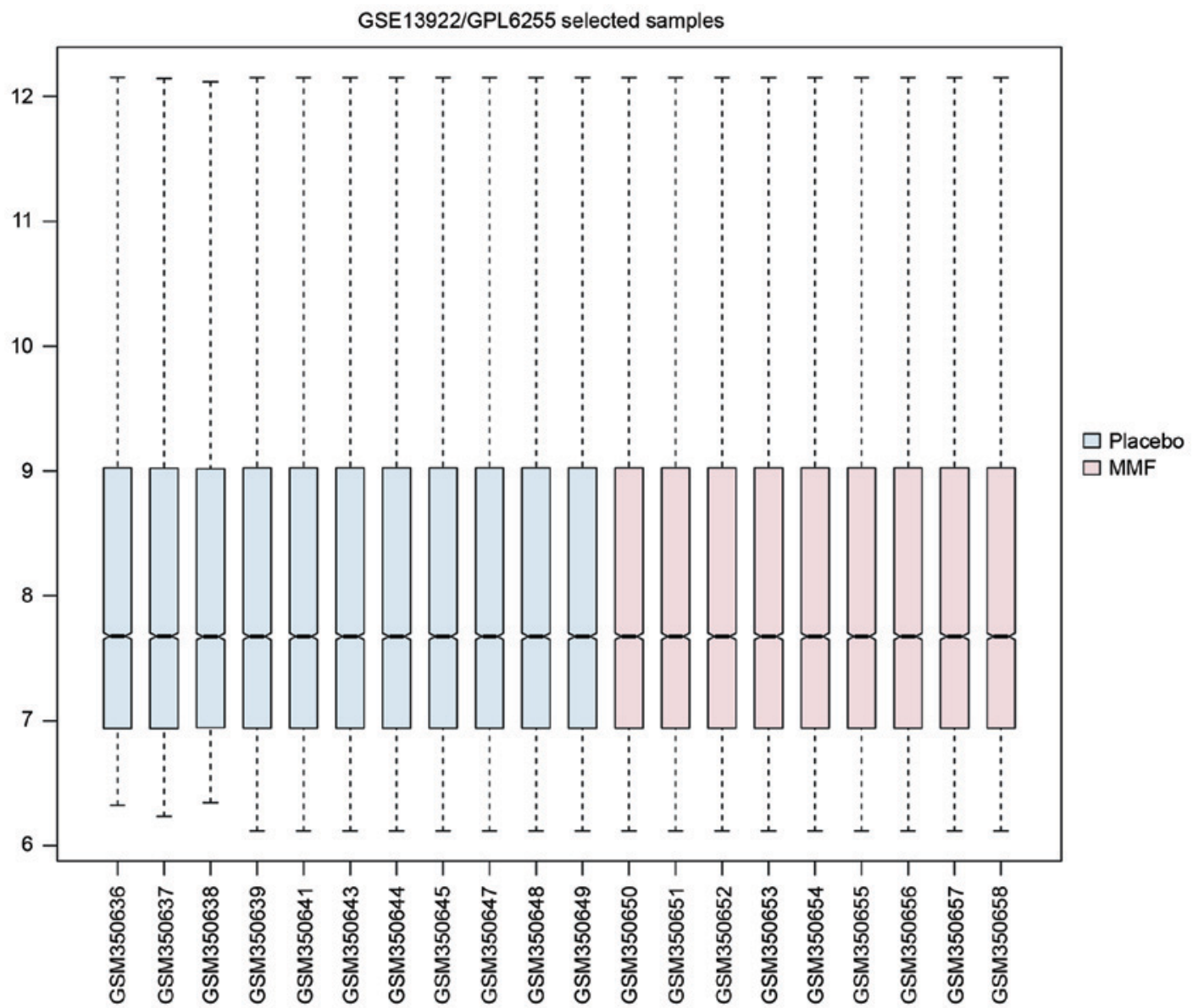

Figure 1. Box plot of the mRNA microarray datasets after normalization. The horizontal axis represents the name of the samples, whereas the vertical axis represents the expression value. Short black lines are used to identify the degree of standardization. MMF, mycophenolate mofetil. 


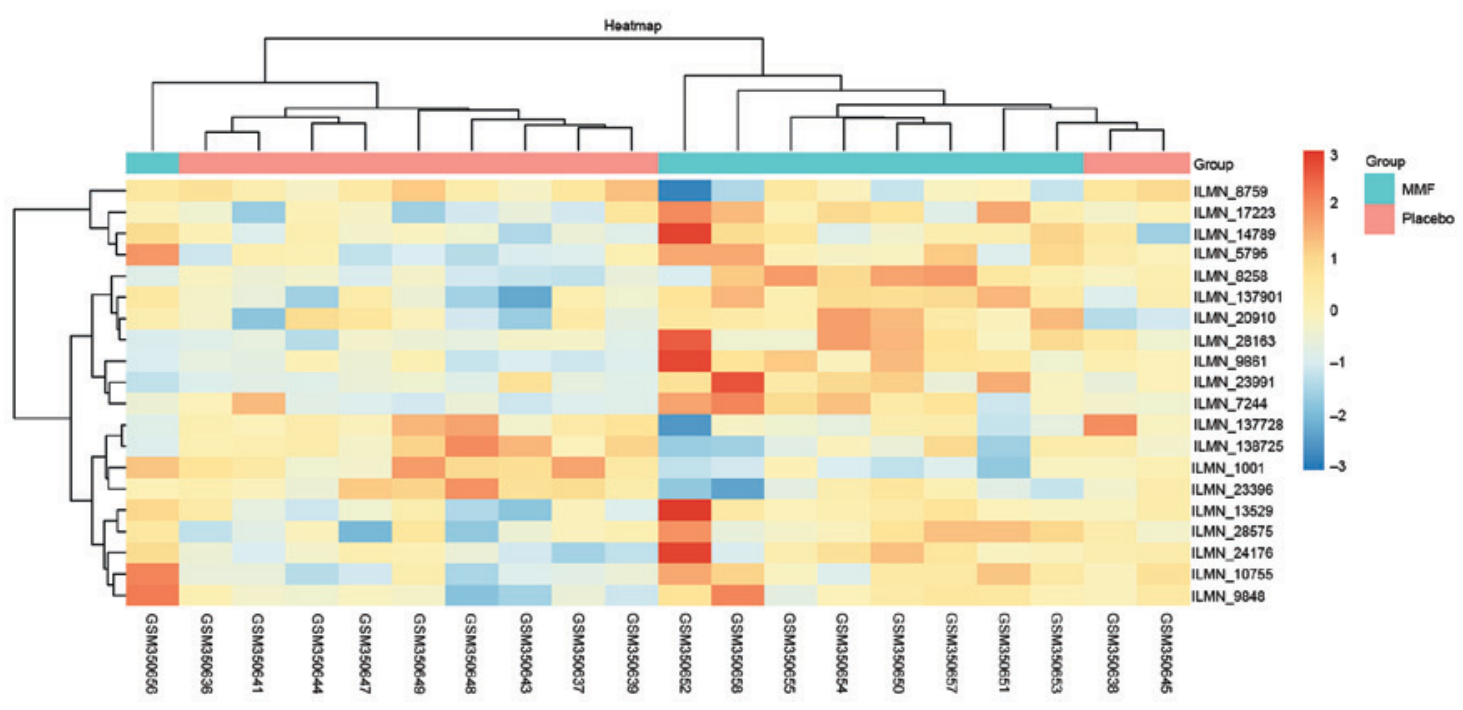

Figure 2. Hierarchical clustering dendrogram of gene expression. A total of 19 DEGs were screened in carotid endarterectomy samples from 11 MMF-treated and 9 placebo-treated patients with symptomatic coronary artery stenosis. A change in color from red to blue indicated high to low expression. DEGs, differentially-expressed genes; MMF, mycophenolate mofetil.

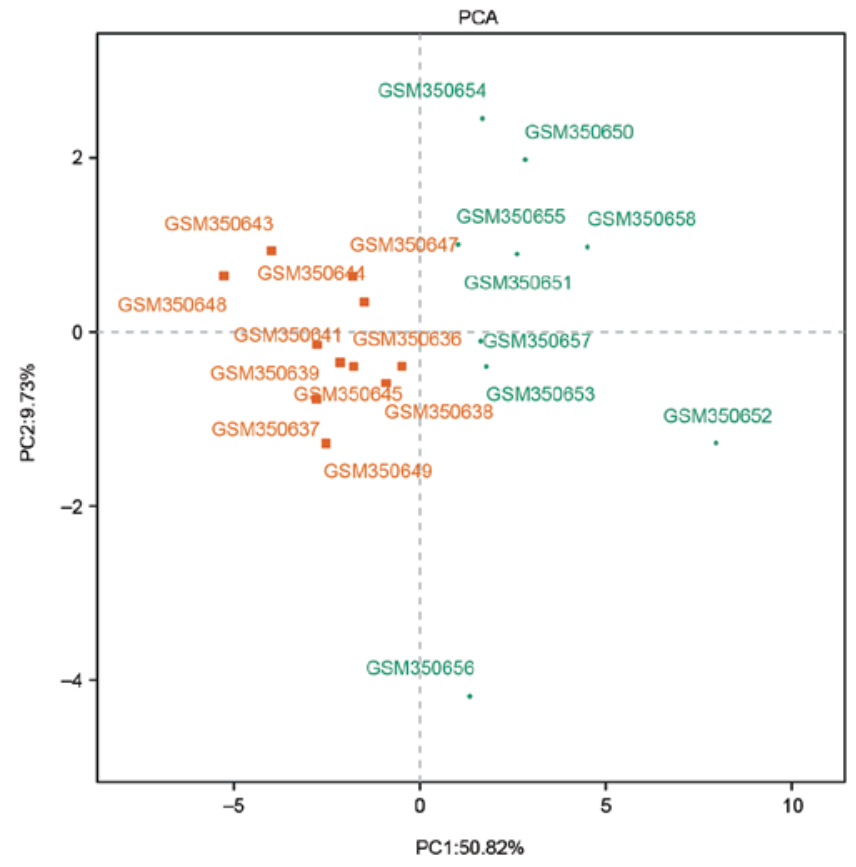

Figure 3. PCA of the 19 differentially-expressed genes from 20 samples The horizontal axis represents the scores of first principal components and the vertical axis represents the scores of second principal components of samples. In the first principal components, $50.82 \%$ of variances were explained, whereas in the second principal component, $9.73 \%$ of variances were explained. Totally, the resolution degree of variances was $60.55 \%$. PCA, principal components analysis.

the pairs isolated from the major network, the PPI network composed of 172 edges and 105 nodes (Fig. 4). In the network, nodes represent genes and edges represent the interaction between genes. The PPI network of DEGs was in a state of high aggregation, which is an essential property of biological networks. With a threshold degree $\geq 3$, a total of 43 genes were selected (Table II). As shown in Fig. 4, tumor protein p53 (degree, 33) and E1A binding protein P300 (degree, 18) had more degrees than the other DEGs.
GO functional analysis. Using a threshold of $\mathrm{P}<0.05$, a total of 401 significant GO terms were enriched and the top 10 enriched terms for each category are presented in Fig. 5; only 8 terms were enriched in cellular components. The most enriched GO terms of the DEGs were mainly associated with tyrosine phosphorylation of signal transducer and activator of transcription-5 (Stat5) protein, which is closely associated with the activation of $\mathrm{T}$ cells.

KEGG pathway enrichment analysis. Using a threshold of $\mathrm{P}<0.05$, a total of 6 significant KEGG pathways were enriched (Fig. 6). The most enriched KEGG pathways of the DEGs were mainly associated with the pharmacological functioning of MMF in activated $\mathrm{T}$ cells, including purine metabolism, glutathione metabolism and pyrimidine metabolism.

\section{Discussion}

The treatment of CAS includes three modalities: Medical management, carotid artery angioplasty and stenting, and CEA (26). However, there are doubts regarding the efficacy of carotid artery angioplasty and stenting, and CEA, in specific patients (27-29). Previous studies have aimed to identify novel treatments and medications for atherosclerosis. Following construction of the hypercholesterolaemic apolipoprotein E-deficient murine model, Chen et al (30) demonstrated that preimplantation factor could prevent atherosclerosis via its immunomodulatory effects without affecting serum lipids. In addition, Sun et al (31) developed trifunctional Simian virus 40 (SV40)-based nanoparticles for in vivo targeting and imaging of atherosclerotic plaques, and targeted SV40 virus-like nanoparticles were revealed to deliver a greater concentration of the anticoagulant drug Hirulog to atherosclerotic plaques.

It is well known that MMF is a T cell suppressor, which is able to reduce synthesis of guanine via IMPDH inhibition, resulting in the suppression of $\mathrm{T}$ cell proliferation (32). van Leuven et al (19) demonstrated that treatment with 
Table II. DEGs with degree $\geq 3$.

\begin{tabular}{|c|c|c|c|c|}
\hline Gene symbol & Gene name & P-value & $\log \mathrm{FC}$ & Degree \\
\hline$\underline{\mathrm{CKB}}$ & Creatine kinase B & 0.0011 & 0.957 & 3 \\
\hline$\underline{\text { KIT }}$ & KIT proto-oncogene receptor tyrosine kinase & 0.0018 & 0.6283 & 10 \\
\hline AK4 & Adenylate kinase 4 & 0.0042 & 0.4736 & 7 \\
\hline POLR2I & RNA polymerase II subunit I & 0.0053 & 0.2292 & 4 \\
\hline$\underline{\text { APLN }}$ & Apelin & 0.0092 & 0.5261 & 3 \\
\hline RRM1 & Ribonucleotide reductase catalytic subunit M1 & 0.0094 & 0.2653 & 7 \\
\hline CSF2 & Colony stimulating factor 2 & 0.0097 & 0.2979 & 7 \\
\hline ENO2 & Enolase 2 & 0.0143 & 0.5576 & 4 \\
\hline NUP98 & Nucleoporin 98 & 0.0174 & 0.1405 & 5 \\
\hline TLR4 & Toll like receptor 4 & 0.0179 & -0.422 & 8 \\
\hline NES & Nestin & 0.0189 & 0.481 & 3 \\
\hline CD2BP2 & CD2 cytoplasmic tail binding protein 2 & 0.019 & 0.1659 & 3 \\
\hline PPP3CA & Protein phosphatase 3 catalytic subunit $\alpha$ & 0.019 & 0.2495 & 5 \\
\hline COPS6 & COP9 signalosome subunit 6 & 0.0196 & 0.2328 & 4 \\
\hline FST & Follistatin & 0.0199 & 0.7444 & 3 \\
\hline EP300 & E1A binding protein p300 & 0.0202 & 0.2351 & 18 \\
\hline BBS 10 & Bardet-Biedl syndrome 10 & 0.0211 & 0.2222 & 5 \\
\hline MAP1B & Microtubule associated protein $1 \mathrm{~B}$ & 0.0215 & 0.1588 & 3 \\
\hline HNRNPA3 & Heterogeneous nuclear ribonucleoprotein A3 & 0.022 & 0.2015 & 5 \\
\hline PDE4D & Phosphodiesterase 4D & 0.023 & 0.2095 & 4 \\
\hline SOD2 & Superoxide dismutase 2 , mitochondrial & 0.0236 & 0.3115 & 5 \\
\hline PRKDC & Protein kinase, DNA-activated, catalytic polypeptide & 0.0252 & 0.3726 & 6 \\
\hline TFDP2 & Transcription factor Dp-2 & 0.026 & 0.232 & 5 \\
\hline GPX1 & Glutathione peroxidase 1 & 0.0261 & -0.7343 & 4 \\
\hline$\overline{\text { PTGES }}$ & Prostaglandin E synthase & 0.0275 & 0.2743 & 3 \\
\hline ACO1 & Aconitase 1 & 0.0303 & 0.3555 & 7 \\
\hline RAN & RAN, member RAS oncogene family & 0.0306 & 0.3282 & 3 \\
\hline COL5A3 & Collagen type $\mathrm{V} \alpha 3$ chain & 0.0316 & 0.1173 & 3 \\
\hline USF1 & Upstream transcription factor 1 & 0.0332 & -0.4322 & 3 \\
\hline SOX9 & SRY-box 9 & 0.0345 & 0.2414 & 7 \\
\hline PDE2A & Phosphodiesterase 2A & 0.0356 & 0.1566 & 3 \\
\hline CHMP2A & Charged multivesicular body protein $2 \mathrm{~A}$ & 0.0361 & 0.1974 & 3 \\
\hline AK8 & Adenylate kinase 8 & 0.0362 & 0.2411 & 8 \\
\hline DHX9 & DEAH-box helicase 9 & 0.0367 & 0.1559 & 5 \\
\hline LEP & Leptin & 0.0373 & 0.7399 & 7 \\
\hline$\overline{\mathrm{TP} 53}$ & Tumor protein $\mathrm{p} 53$ & 0.038 & 0.1518 & 33 \\
\hline YWHAE & $\begin{array}{l}\text { Tyrosine } 3 \text {-monooxygenase/tryptophan 5-monooxygenase } \\
\text { Activation protein epsilon }\end{array}$ & 0.0383 & 0.1716 & 5 \\
\hline MCM4 & Minichromosome maintenance complex component 4 & 0.0393 & 0.2495 & 5 \\
\hline PFKP & Phosphofructokinase, platelet & 0.0395 & 0.3782 & 4 \\
\hline LMNA & Lamin $\mathrm{A} / \mathrm{C}$ & 0.0446 & 0.4168 & 5 \\
\hline СCT3 & Chaperonin containing TCP 1 subunit 3 & 0.0446 & 0.2518 & 5 \\
\hline BMP2 & Bone morphogenetic protein 2 & 0.0458 & 0.1583 & 11 \\
\hline DYRK1A & $\begin{array}{l}\text { Dual specificity tyrosine phosphorylation } \\
\text { Regulated kinase } 1 \mathrm{~A}\end{array}$ & 0.0495 & -0.2047 & 5 \\
\hline
\end{tabular}

FC, fold change. Gene symbols shown in bold represent genes with $\mathrm{P}<0.01$ and underlined genes represent the gene with llog fold changel $\geq 0.5$.

MMF was able to reduce inflammatory cell infiltration, with a concomitant decrease in proinflammatory gene expression in patients with SCAS. The present study downloaded and analyzed a GEO mRNA expression profile uploaded by van Leuven et al (19). A total of 210 DEGs were identified between MMF-treated and placebo-treated groups. 
Table III. Most significantly enriched GO terms $(\mathrm{P}<0.001)$.

\begin{tabular}{lll}
\hline GO ID & \multicolumn{1}{c}{ GO term } & \multicolumn{1}{c}{ Associated genes } \\
\hline GO:0048513 & Organ development & AK4, APLN, CKB, CSF2, ECE1, GPM6B, KIT, \\
GO:0048731 & System development & LMO4, NSDHL, SCIN \\
& & AK4, APLN, CKB, CSF2, ECE1, GFRA2, \\
GO:0042523 & $\begin{array}{l}\text { Positive regulation of tyrosine } \\
\text { phosphorylation of Stat5 protein } \\
\text { Regulation of tyrosine phosphorylation } \\
\text { of Stat5 protein }\end{array}$ & GPM6B, KIT, LMO4, NSDHL, SCIN \\
GO:0042506 & $\begin{array}{l}\text { Tyrosine phosphorylation of Stat5 protein } \\
\text { Megakaryocyte differentiation }\end{array}$ & CSF2, KIT \\
GO:0030219 & Anatomical structure development & CSF2, KIT \\
GO:0048856 & & CSF2, KIT \\
GO:0007275 & Multicellular organismal development & KIT, SCIN \\
GO:0044087 & Regulation of cellular component biogenesis & AK4, APLN, CKB, CSF2, ECE1, GFRA2, \\
GO:0034311 & Diol metabolic process & GPM6B, KIT, LMO4, NSDHL, SCIN \\
\hline & & AK4, APLN, CKB, CSF2, ECE1, GFRA2, \\
& & GPM6B, KIT, LMO4, NSDHL, SCIN
\end{tabular}

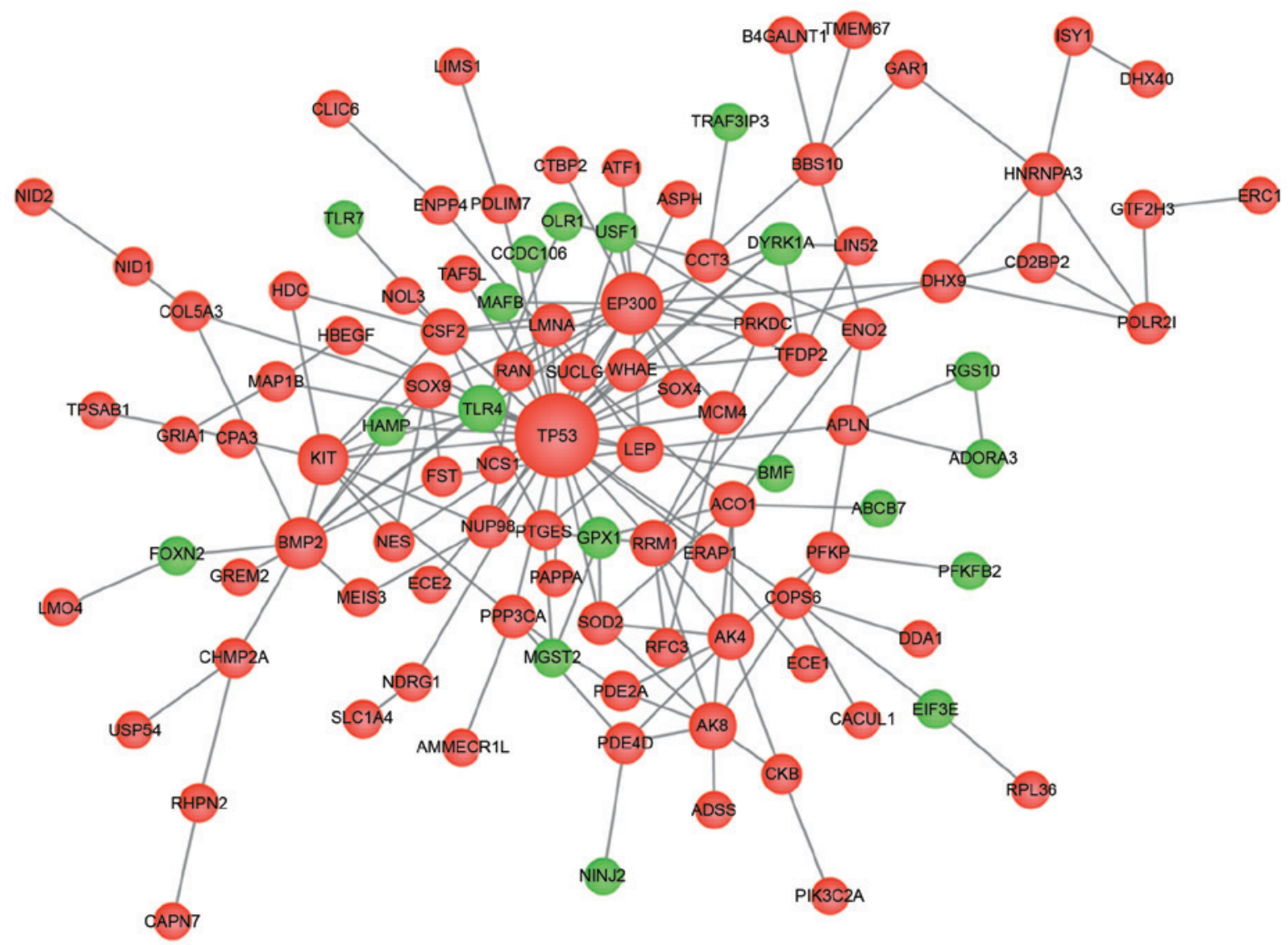

Figure 4. Protein-protein interaction network of DEGs. Red nodes represent upregulated DEGs, and green nodes represent downregulated DEGs; the connecting lines represent the interactions between DEGs. The size of the node is proportional to the degree of the DEG. DEGs, differentially-expressed genes.

Subsequently, the 19 most significant DEGs were selected to undergo GO functional analysis and KEGG pathway enrichment analysis. The results revealed that the most enriched
KEGG pathway was purine metabolism, indicating that suppression of inflammatory activity served an important role in the MMF treatment of patients with SCAS. Furthermore, 


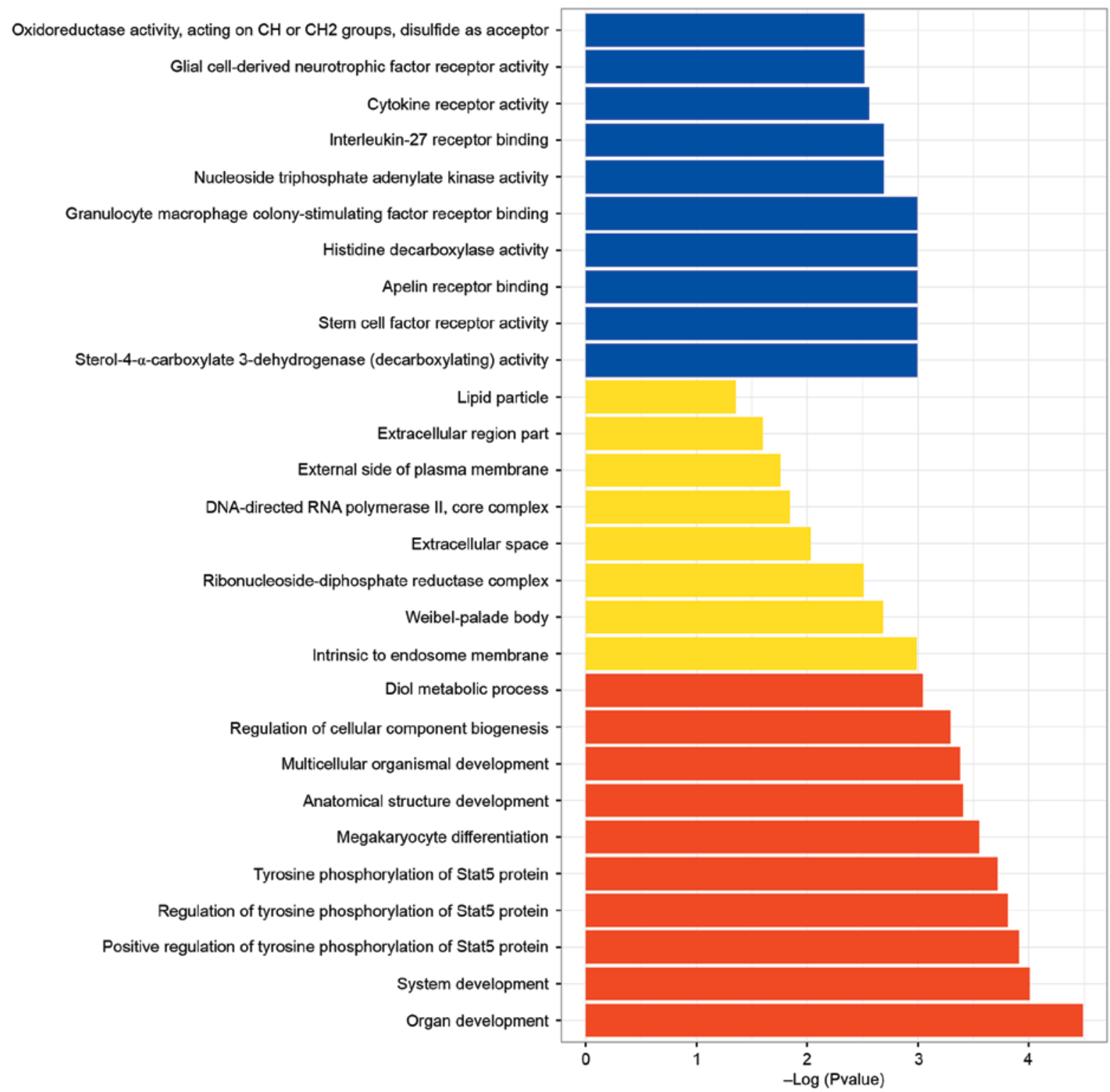

Figure 5. GO term analysis, separated into biological process, cellular component and molecular function categories, for differentially-expressed genes. Top 10 enriched terms of each category are shown; only 8 terms are presented for cellular components. Red bars represent biological process terms; yellow bars represent cell component terms; blue bars represent molecular function terms. The vertical axis represents the GO category and the horizontal axis represents the - $\log$ (p-value). $\mathrm{P}<0.05$ was used as the threshold for selecting significant GO categories. GO, Gene Ontology.

with a threshold of degree $\geq 3$ and $\log \mathrm{FCl} \geq 0.5$, three genes, APLN, creatine kinase B (CKB) and KIT, were selected as the key genes.

APLN is a peptide, which was initially identified by Tatemoto et al in 1998 (33), that functions as an endogenous ligand for the orphaned G-protein-coupled receptor (APJ) (34). Previous studies have revealed that APJ deficiency can prevent oxidative stress-associated atherosclerosis and that the APLN-APJ system is a mediator of oxidative stress in vascular tissue (33,35-37). In the present study, expression of APLN was significantly different between MMF-treated and placebo-treated groups, and APLN was a key gene in the PPI network, indicating that APLN may be a target of MMF for the treatment of SCAS. According to the results of the KEGG analysis, purine metabolism was the most enriched signaling pathway, indicating that MMF modified the atherosclerotic plaque by suppressing activated $\mathrm{T}$ cells.
Recent studies have also reported that APLN is involved in the immune response $(38,39)$. By analyzing the expression and function of the APLN-APJ system in tumor vasculature, Kidoya et al (38) indicated that the APLN-APJ system could induce maturation of tumor vasculature and improve the efficiency of immune therapy. The results of the GO analysis suggested that terms associated with Stat5 were the most enriched, including tyrosine phosphorylation of Stat5 protein, regulation of tyrosine phosphorylation of Stat5 protein and positive regulation of tyrosine phosphorylation of Stat5 protein (Table III). Previous studies have indicated that Stat5 has a strong association with T cells. Lindahl et al (40) provided evidence to suggest that microRNA-21 is expressed in situ in cutaneous $\mathrm{T}$ cell lymphomas skin lesions, as induced by IL-2 and IL- 15 cytokines, and is regulated by Stat5 in malignant T cells. The APLN-APJ system is also involved in the immune response and Stat3. Han et al (41) indicated 


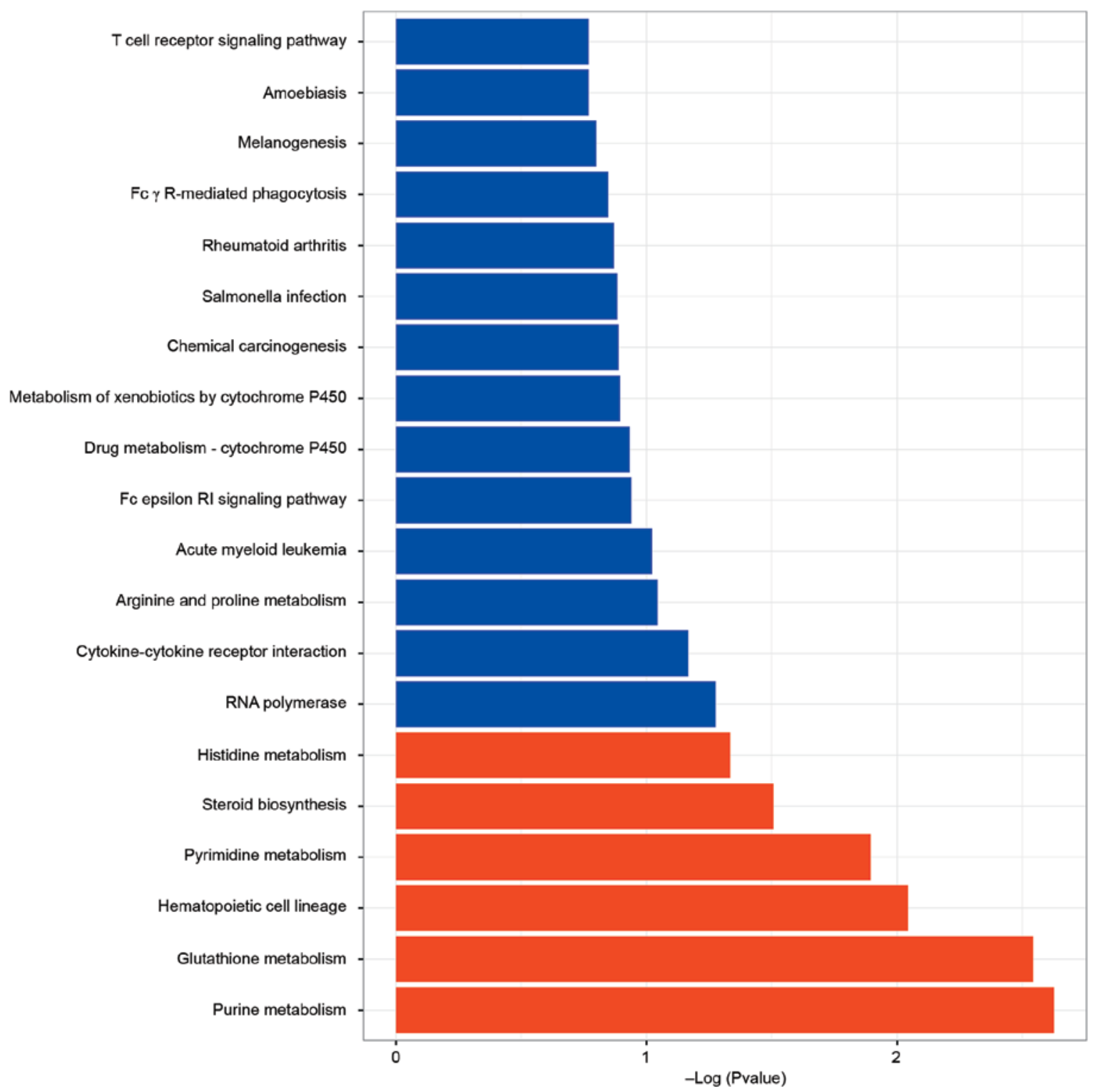

Figure 6. KEGG pathway analysis for differentially-expressed genes. The horizontal axis represents the -log (p-value) of the pathway and the vertical axis represents the pathway list. $\mathrm{P}<0.05$ was used as the threshold for selecting the significant KEGG pathways (red bars). KEGG, Kyoto Encyclopedia of Genes and Genomes.

that binding of phosphorylated-Stat 3 to the APLN promoter is the final step underlying proinflammatory cytokine-induced enteric APLN expression during intestinal inflammation. However, whether APLN is involved in the Stat5 signaling remains to be elucidated. It may be hypothesized that APLN affects T cells through the Stat5 signaling pathway; however, further studies are required.

Mast/stem cell growth factor receptor, also known as proto-oncogene c-Kit, tyrosine-protein kinase Kit, or cluster of differentiation 117 is a receptor tyrosine kinase protein that in humans is encoded by the KIT gene (42). The GO analysis results revealed that KIT was closely associated with tyrosine phosphorylation of Stat5 protein, thus indicating that dysregulation of Stat5 may be important in the process of SCAS.

Brain-type creatine kinase is a creatine kinase encoded by the CKB gene in humans, which is associated with creatine kinase activity and cellular monovalent inorganic anion homeostasis (43). However, to the best of our knowledge, the involvement of CKB in the process of SCAS has not yet been reported.

In conclusion, APLN and KIT may serve important roles in the MMF treatment of SCAS. The results of the present study suggested that MMF may upregulate APLN to inhibit the proliferation of $\mathrm{T}$ cells through the Stat5 signaling pathway. Further investigation of the function of APLN and KIT in atherosclerosis is urgently required.

\section{Acknowledgements}

The present study was supported by the Youth Innovation Fund Projects of Inner Mongolia Medical University (grant no. YKD2013QNCX023), the Health Department Medical Scientific Research Projects of the Inner Mongolia Autonomous Region (grant no. 201302089) and the Health Department Medical Scientific Research Projects of the Inner Mongolia Autonomous Region (grant no. 201302090). 


\section{References}

1. Murphy SL, Xu J and Kochanek KD: Deaths: Final data for 2010 National vital statistics reports: From the Centers for Disease Control and Prevention, National Center for Health Statistics, national vital statistics system 61: 1-117, 2013.

2. Kochanek KD, Xu J, Murphy SL, Minino AM and Kung HC: Deaths: Final data for 2009. National vital statistics reports: From the Centers for Disease Control and Prevention, National Center for Health Statistics, national vital statistics system 59: $1-116,2011$.

3. Grotta JC: Carotid stenosis. N Engl J Med 369: 2360-2361, 2013.

4. Mozaffarian D, Benjamin EJ, Go AS, Arnett DK, Blaha MJ, Cushman M, de Ferranti S, Després JP, Fullerton HJ, Howard VJ, et al: Heart disease and stroke statistics-2015 update: A report from the American Heart Association. Circulation 131: e29-322, 2015.

5. Sacco RL, Adams R, Albers G, Alberts MJ, Benavente O, Furie K, Goldstein LB, Gorelick P, Halperin J, Harbaugh R, et al: Guidelines for prevention of stroke in patients with ischemic stroke or transient ischemic attack: A statement for healthcare professionals from the American Heart Association/American Stroke Association Council on Stroke: Co-sponsored by the Council on Cardiovascular Radiology and Intervention: The American Academy of Neurology affirms the value of this guideline. Circulation 37: e409-e449, 2006.

6. Kistler JP and Furie KL: Carotid endarterectomy revisited. N Engl J Med 342: 1743-1745, 2000.

7. Davies MJ and Thomas A: Thrombosis and acute coronary-artery lesions in sudden cardiac ischemic death. N Engl J Med 310: $1137-1140,1984$

8. Li Z, Hou P, Fan D, Dong M, Ma M, Li H, Yao R, Li Y, Wang G, Geng P, et al: The degradation of EZH2 mediated by lncRNA ANCR attenuated the invasion and metastasis of breast cancer. Cell Death Differ 24: 59-71, 2017.

9. Libby P: Inflammation in atherosclerosis. Arterioscler Thromb Vasc Biol 32: 2045-2051, 2012.

10. Rocha VZ and Libby P: Obesity, inflammation, and atherosclerosis. Nat Rev Cardiol 6: 399-409, 2009.

11. Sparrow CP and Olszewski J: Cellular oxidation of low density lipoprotein is caused by thiol production in media containing transition metal ions. J Lipid Res 34: 1219-1228, 1993.

12. Topper JN and Gimbrone MA Jr: Blood flow and vascular gene expression: Fluid shear stress as a modulator of endothelial phenotype. Mol Med Today 5: 40-46, 1999.

13. De Caterina R,Libby P,Peng HB, Thannickal VJ, RajavashisthTB, Gimbrone MA Jr, Shin WS and Liao JK: Nitric oxide decreases cytokine-induced endothelial activation. Nitric oxide selectively reduces endothelial expression of adhesion molecules and proinflammatory cytokines. J Clin Invest 96: 60-68, 1995.

14. Castellani ML,Anogeianaki A,Felaco P, Toniato E, De Lutiis MA, Shaik B, Fulcheri M, Vecchiet J, Tetè S, Salini V, et al: IL-35, an anti-inflammatory cytokine which expands CD $4^{+} \mathrm{CD} 25^{+}$Treg Cells. J Biol Regul Homeost Agents 24: 131-135, 2010.

15. Oršolić N: Bee venom in cancer therapy. Cancer Metastasis Rev 31: 173-194, 2012.

16. Ma Y, Liu X, Wei Z, Wang X, Xu D, Dai S, Li Y, Gao M, Ji C, Guo C, et al: The expression of a novel anti-inflammatory cytokine IL-35 and its possible significance in childhood asthma. Immunol Lett 162: 11-17, 2014.

17. Huang Y, Lin YZ, Shi Y and Ji QW: IL-35: A potential target for the treatment of atherosclerosis. Pharmazie 68: 793-795, 2013.

18. Allison AC and Eugui EM: Mechanisms of action of mycophenolate mofetil in preventing acute and chronic allograft rejection. Transplantation 80 (2 Suppl): S181-S190, 2005.

19. van Leuven SI, van Wijk DF, Volger OL, de Vries JP, van der Loos CM, de Kleijn DV, Horrevoets AJ, Tak PP, van der Wal AC, de Boer OJ, et al: Mycophenolate mofetil attenuates plaque inflammation in patients with symptomatic carotid artery stenosis. Atherosclerosis 211: 231-236, 2010.

20. Irizarry RA, Bolstad BM, Collin F, Cope LM, Hobbs B and Speed TP: Summaries of Affymetrix GeneChip probe level data. Nucleic Acids Res 31: e15, 2003.

21. Diboun I, Wernisch L, Orengo CA and Koltzenburg M Microarray analysis after RNA amplification can detect pronounced differences in gene expression using limma. BMC Genomics 7: 252, 2006
22. Ashburner M, Ball CA, Blake JA, Botstein D, Butler $\mathrm{H}$, Cherry JM, Davis AP, Dolinski K, Dwight SS, Eppig JT, et al: Gene ontology: Tool for the unification of biology. The gene ontology consortium. Nat Genet 25: 25-29, 2000.

23. Kanehisa M and Goto S: KEGG: Kyoto encyclopedia of genes and genomes. Nucleic Acids Res 28: 27-30, 2000.

24. Huang da W, Sherman BT, Lempicki RA: Systematic and integrative analysis of large gene lists using DAVID bioinformatics resources. Nat Protoc 4: 44-57, 2009.

25. Hosack DA, Dennis G Jr, Sherman BT, Lane HC and Lempicki RA: Identifying biological themes within lists of genes with EASE. Genome Biol 4: R70, 2003.

26. Bae C, Szuchmacher M and Chang JB: Comparative review of the treatment methodologies of carotid stenosis. Int J Angiol 24: 215-222, 2015.

27. Tsukahara T, Hatano T, Nakakuki T, Tsuji Y, Aoyama T and Ogata H: Combined treatment using CEA and CAS for carotid arterial stenosis. Acta Neurochir Suppl 103: 109-112, 2008.

28. De Borst GJ: Recruiting RCTs comparing CAS, CEA and best medical treatment for asymptomatic carotid stenosis. J Cardiovasc Surg (Torino) 56: 837-844, 2015.

29. Lal BK: Recurrent carotid stenosis after CEA and CAS: Diagnosis and management. Semin Vasc Surg 20: 259-266, 2007.

30. Chen YC, Rivera J, Fitzgerald M, Hausding C, Ying YL, Wang X, Todorova K, Hayrabedyan S, Barnea ER and Peter K: PreImplantation factor prevents atherosclerosis via its immunomodulatory effects without affecting serum lipids. Thromb Haemost 115: 1010-1024, 2016.

31. Sun X, Li W, Zhang X, Qi M, Zhang Z, Zhang XE and Cui Z: In Vivo targeting and imaging of atherosclerosis using multifunctional virus-like particles of simian virus 40. Nano Lett 16 6164-6171, 2016.

32. Allison AC and Eugui EM: Purine metabolism and immunosuppressive effects of mycophenolate mofetil (MMF). Clin Transplant 10: 77-84, 1996.

33. Tatemoto K, Hosoya M, Habata Y, Fujii R, Kakegawa T, Zou MX, Kawamata Y, Fukusumi S, Hinuma S, Kitada C, et al: Isolation and characterization of a novel endogenous peptide ligand for the human APJ receptor. Biochem Biophys Res Commun 251: 471-476, 1998.

34. Falcão-Pires I and Leite-Moreira AF: Apelin: A novel neurohumoral modulator of the cardiovascular system. Pathophysiologic importance and potential use as a therapeutic target. Rev Port Cardiol 24: 1263-1276, 2005.

35. Kleinz MJ, Skepper JN and Davenport AP: Immunocy tochemical localisation of the apelin receptor, APJ, to human cardiomyocytes, vascular smooth muscle and endothelial cells. Regul Pept 126: 233-240, 2005.

36. Kleinz MJ and Davenport AP: Immunocytochemical localization of the endogenous vasoactive peptide apelin to human vascular and endocardial endothelial cells. Regul Pept 118: 119-125, 2004.

37. Hashimoto T, Kihara M, Imai N, Yoshida S, Shimoyamada $H$, Yasuzaki H, Ishida J, Toya Y, Kiuchi Y, Hirawa N, et al: Requirement of apelin-apelin receptor system for oxidative stress-linked atherosclerosis. Am J Pathol 171: 1705-1712, 2007.

38. Kidoya H, Kunii N, Naito H, Muramatsu F, Okamoto Y, Nakayama T and Takakura N: The apelin/APJ system induces maturation of the tumor vasculature and improves the efficiency of immune therapy. Oncogene 31: 3254-3264, 2012.

39. Adam F, Khatib AM, Lopez JJ, Vatier C, Turpin S, Muscat A, Soulet F, Aries A, Jardin I,Bobe R, et al: Apelin: An antithrombotic factor that inhibits platelet function. Blood 127: 908-920, 2016.

40. Lindahl LM, Fredholm S, Joseph C, Nielsen BS, Jønson L, Willerslev-Olsen A, Gluud M, Blümel E, Petersen DL, Sibbesen N, et al: STAT5 induces miR-21 expression in cutaneous T cell lymphoma. Oncotarget 7: 45730-45744, 2016.

41. Han S, Wang G, Qi X, Englander EW and Greeley GH Jr: Involvement of a Stat 3 binding site in inflammation-induced enteric apelin expression. Am J Physiol Gastrointest Liver Physiol 295: G1068-G1078, 2008.

42. Andre C, Hampe A, Lachaume P, Martin E, Wang XP, Manus V, $\mathrm{Hu}$ WX and Galibert F: Sequence analysis of two genomic regions containing the KIT and the FMS receptor tyrosine kinase genes. Genomics 39: 216-226, 1997.

43. Mariman EC, Schepens JT and Wieringa B: Complete nucleotide sequence of the human creatine kinase B gene. Nucleic Acids Res 17: 6385, 1989. 